\title{
Retroperitoneal Neurofibroma and a Malignant Peripheral Nerve Sheath Tumor with Neurofibromatosis Type 1: A Report of Two Cases
}

\author{
Kumiko Yotsuya, Tomohiko Hasegawa, Yu Yamato, Go Yoshida, Tatsuya Yasuda, Tomohiro Banno, Hideyuki Arima, \\ Shin Oe and Yukihiro Matsuyama
}

Department of Orthopedic Surgery, Hamamatsu University School of Medicine, Hamamatsu, Japan

\author{
Keywords: \\ malignant peripheral nerve sheath tumor (MPNST), neurofibroma, neurofibromatosis type 1 (NF1) \\ Spine Surg Relat Res 2020; 4(4): 369-373 \\ dx.doi.org/10.22603/ssrr.2020-0056
}

Malignant peripheral nerve sheath tumors (MPNSTs) are highly aggressive sarcomas, with an incidence of $0.001 \%$ in the general population compared with $2 \%-5 \%$ in patients with neurofibromatosis type $1(\mathrm{NF} 1)^{1)}$. In patients with NF1, the life-time risk of MPNST is $8 \%-13 \%$, and MPNSTs frequently cause death ${ }^{2}$. Approximately $40 \%$ MPNSTs occur at deep locations ${ }^{2}$; differentiating MPNSTs from neurofibromas in deep lesions of patients with NF1 is important. We report two cases of huge retroperitoneal tumors with NF-1. Although the background and tumor progression of the cases were similar, the diagnosis was MPNST and neurofibroma. This report compares images in both cases and discusses the magnetic resonance imaging (MRI) findings suggesting MPNSTs.

Case 1 involved a 44-year-old man with NF1 who presented with left lower limb pain for a month. Physical examination revealed no muscle weakness or sensory disturbance. MRI showed a $5 \times 7 \times 11 \mathrm{~cm}$ lesion extending from the left L4 nerve root to the retroperitoneum (Fig. 1). T1weighted image (T1WI) showed a low signal, and T2weighted image (T2WI) showed heterogeneous low intensity with a high-intense signal area involving a cyst. Gadolinium-enhanced T1WI showed an inhomogeneous enhanced pattern. Computed tomography (CT) showed no calcification. Tumor resection and L4-5 posterior fusion were performed (Fig. 2a-d). The tumor was excised in one piece under the capsule (Fig. 2e). The pain disappeared postsurgery. The tumor presented as a solid lobulated mass, an inner white area with hemorrhage and necrosis, and an outer yellowish-white area formed an intranodal nodule (Fig. 2f).
Pathological findings showed the inside lesion to be an MPNST and the outside area to be a neurofibroma; thus, an MPNST arising from neurofibroma was diagnosed (Fig. 2gi).

Case 2 involved a 44-year-old man with NF1 who presented with right back pain for 3 months. Physical examination revealed no muscle weakness or sensory disturbance. MRI showed a $9 \times 8 \times 6 \mathrm{~cm}$ dumbbell-shaped lesion originating from the L1 nerve root extending from the intraspinal canal to the L1 vertebral body and retroperitoneum (Fig. 3af). The lesion displayed low intensity on T1WI, heterogeneous high intensity on T2WI, and a central faint enhancement pattern with gadolinium. CT revealed an osteolytic lesion with marginal sclerosis in the L1 vertebral body (Fig. 3g-i). Tumor resection and T11-L3 posterior and T12-L2 anterior fusion were performed (Fig. 4a-d). The tumor was excised in one piece under the capsule (Fig. 4e). Pain improved immediately post-surgery. The tumor presented as a uniform yellow-white solid mass without necrosis, diagnosed as neurofibroma (Fig. 4f-h).

Some reports that mentioned MRI being useful for differentiating MPNSTs from neurofibroma. Wasa reported two or more of the four tumoral MRI features - intratumoral cystic lesion, largest dimension $>10 \mathrm{~cm}$, peripheral enhancement pattern, and perilesional edema-like zone-indicated MPNSTs with a sensitivity and specificity of $61 \%$ and $90 \%$, respectively ${ }^{3}$. In case 1 , the tumor presented as $11 \mathrm{~cm}$, exhibited an intratumoral cyst reflecting hemorrhage and necrosis, and was actually diagnosed as MPNST. Lack of a peripheral enhancement pattern and an edema-like zone may 

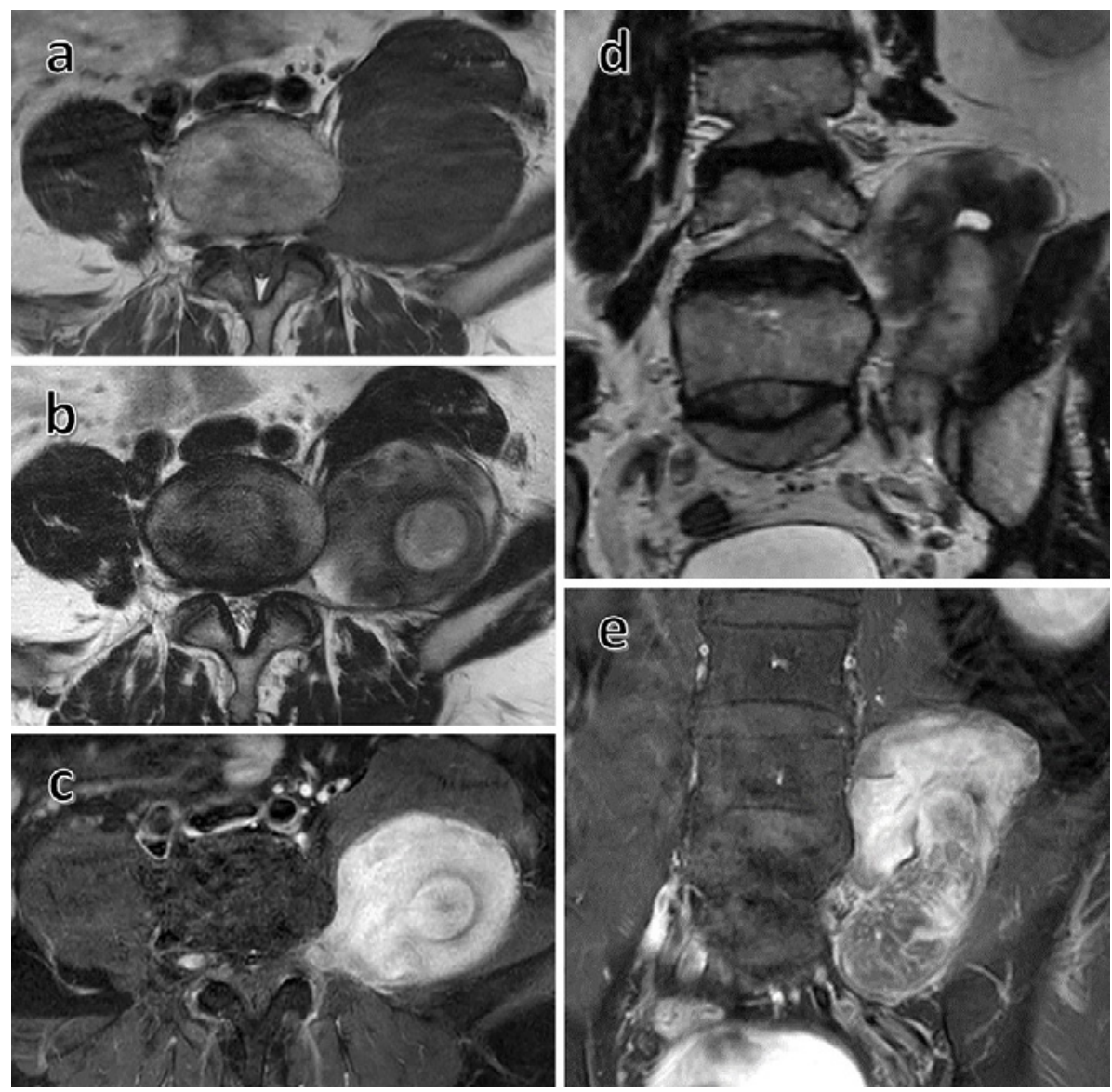

Figure 1. Case 1: A 44-year-old man. Preoperative magnetic resonance imaging (MRI) showed a $5 \times 7 \times 11 \mathrm{~cm}$ lesion extending from the left $\mathrm{L} 4$ nerve root to the retroperitoneum. T1-weighted image (T1WI) showed low intensity, and T2-weighted image (T2WI) showed inhomogeneous low intensity with a high-intense signal area involving a cyst. Gadolinium-enhanced T1WI showed an inhomogeneous enhanced pattern. (a) T1-weighted axial image. (b) T2-weighted axial image. (c) Gadolinium-enhanced T1-weighted axial image. (d) T2-weighted coronal image. (e) Gadolinium-enhanced T1-weighted coronal image.

be due to the MPNST being surrounded by neurofibromas. In case 2, the tumor did not exhibit any of the four features and was actually diagnosed as a neurofibroma. Irregular tumor shape, unclear margin, and intratumoral lobulation were also reported as findings suggesting MPNSTs ${ }^{4}$. Of these, intratumoral lobulation was only seen in case 1 .

MPNSTs surrounded by neurofibromas, as in case 1, may not manifest typical findings suggesting MPNTs such as peripheral enhancement pattern, perilesional edema-like zone, irregular tumor shape, and unclear margin. MRI is helpful for differentiating MPNSTs from neurofibromas, but MPNSTs arising from neurofibromas may require special attention.

Conflicts of Interest: The authors declare that there are no relevant conflicts of interest.

\section{Ethical Approval: None.}

Author Contributions: Kumiko Yotsuya wrote and prepared the manuscript, and all of the authors participated in the study design. All authors read, reviewed, and approved the article.

Informed Consent: Informed consent was obtained from all participants in this study.

\section{References}

1. Ducatman BS, Scheithauer BW, Piepgras DG, et al. Malignant peripheral nerve sheath tumors: a clinicopathological study of 120 patients. Cancer. 1986;57(10):2006-21.

2. Evans DG, Baser ME, McGaughran J, et al. Malignant peripheral nerve sheath tumors in neurofibromatosis 1. J Med Genet. 2002;39 (5):311-4.

3. Wasa J, Nishida Y, Tsukushi S, et al. MRI features in the differentiation of malignant peripheral nerve sheath tumors and neurofibromas. AJR Am J Roentgenol. 2010;194(6):1568-74.

4. Matsumine A, Kusuzaki K, Nakamura T, et al. Differentiation between neurofibromas and malignant peripheral nerve sheath tumors in neurofibromatosis 1 evaluated by MRI. J Cancer Res Clin Oncol. 2009;135(7):891-900. 

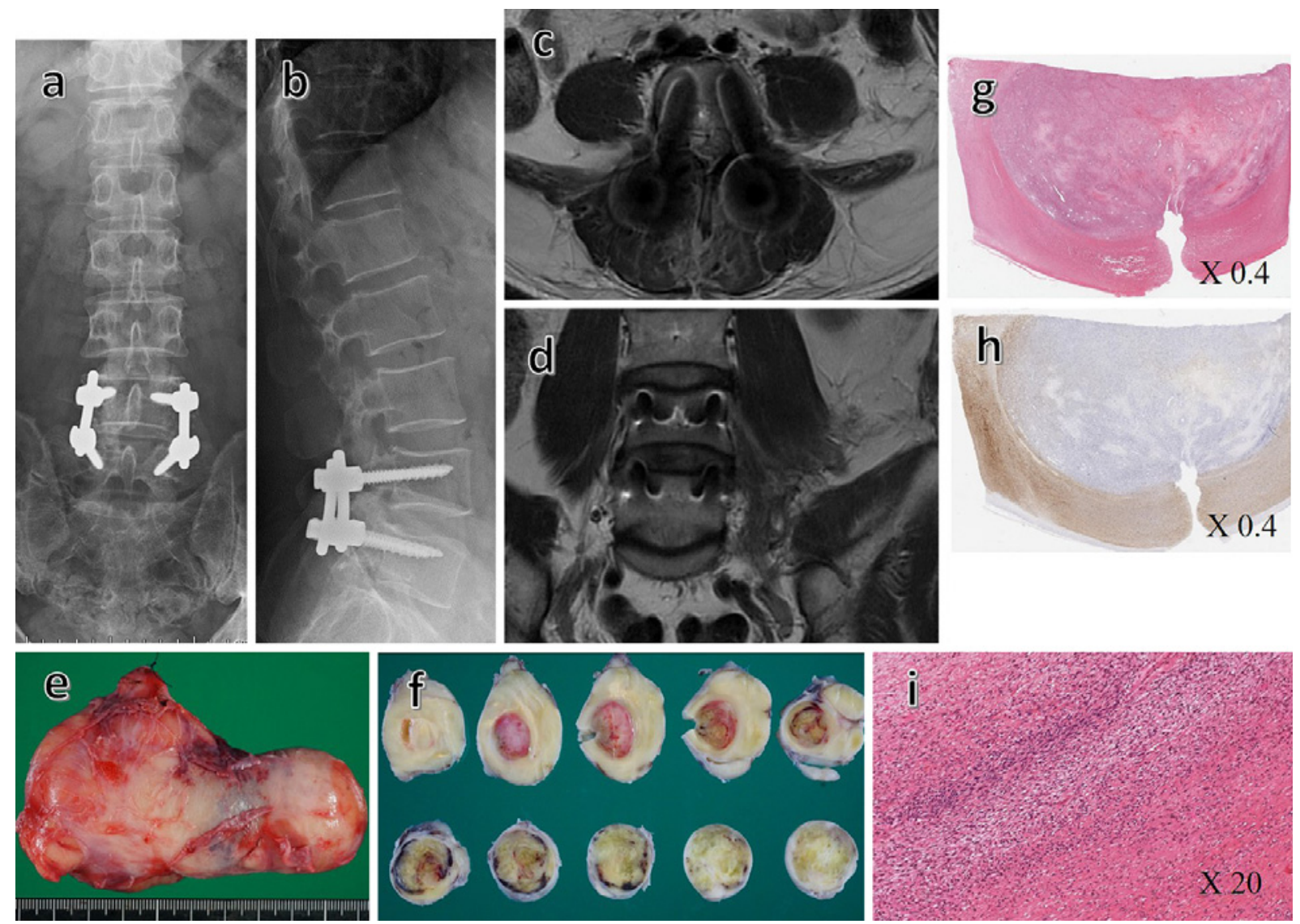

Figure 2. Tumor resection and L4-5 posterior interbody fusion were performed for case 1. (a) Postoperative X-ray, coronal view. (b) Postoperative X-ray, lateral view. (c) Postoperative magnetic resonance imaging. T2-weighted axial image. (d) T2-weighted coronal image. (e) The resected tumor. The tumor was excised in one piece under the capsule. (f) On the cut section, the tumor presented as a solid lobulated mass: an inner white area with hemorrhage and necrosis, and an outer yellowish-white area formed an intranodal nodule. (g) Hematoxylin and eosin staining, 0.4X magnification. (h) S-100 protein immunohistostaining, 0.4X magnification. (i) Hematoxylin and eosin staining, 20X magnification. Outer area shows a proliferation of spindle cells with abundant collagen fibers, showing signs of benign neurofibromas. The inner region exhibits a mixture of findings of atypical neurofibromas with increased spindle cell density and cell atypia and malignant peripheral nerve sheath tumors (MPNSTs) with dense proliferation of atypical spindle cells with necrosis. The histopathological diagnosis was an MPNST arising from a neurofibroma. 

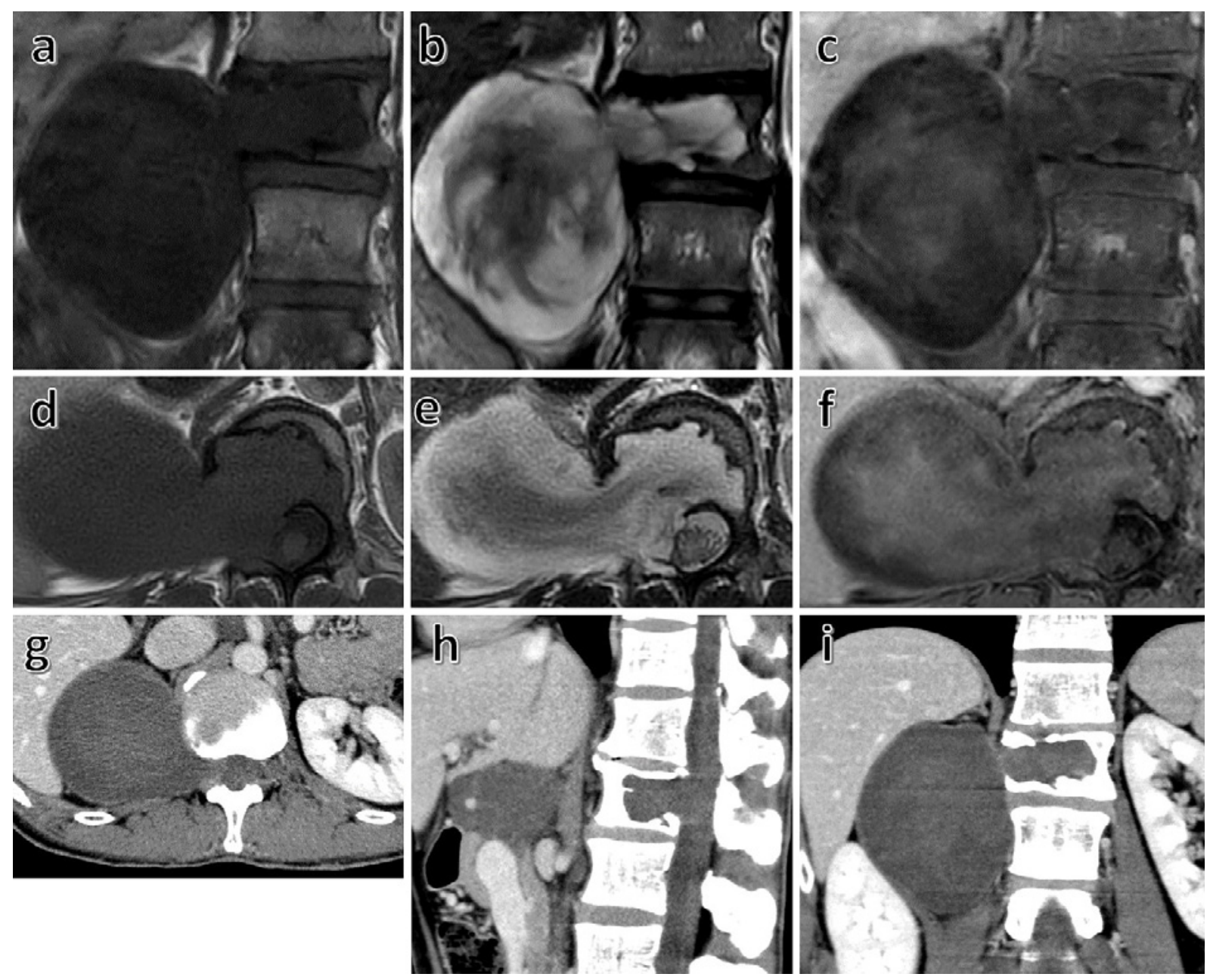

Figure 3. Case 2: A 44-year-old man. Preoperative magnetic resonance imaging showed a 9 × 8 x $6 \mathrm{~cm}$ dumbbell-shaped lesion extending from the L1 vertebral body to the right L1 nerve root and retroperitoneum. The lesion displayed low intensity on T1-weighted image, heterogeneous high intensity on T2-weighted image, and a central faint enhancement pattern with gadolinium. (a) T1-weighted coronal image. (b) T2-weighted coronal image. (c) Gadolinium-enhanced T1-weighted coronal image. (d) T1-weighted axial image. (e) T2-weighted axial image. (f) Gadolinium-enhanced T1-weighted axial image. (g) Preoperative computed tomography (CT) axial view. CT revealed an osteolytic lesion with marginal sclerosis in the L1 vertebral body. (h) CT sagittal view. (i) CT coronal view. 

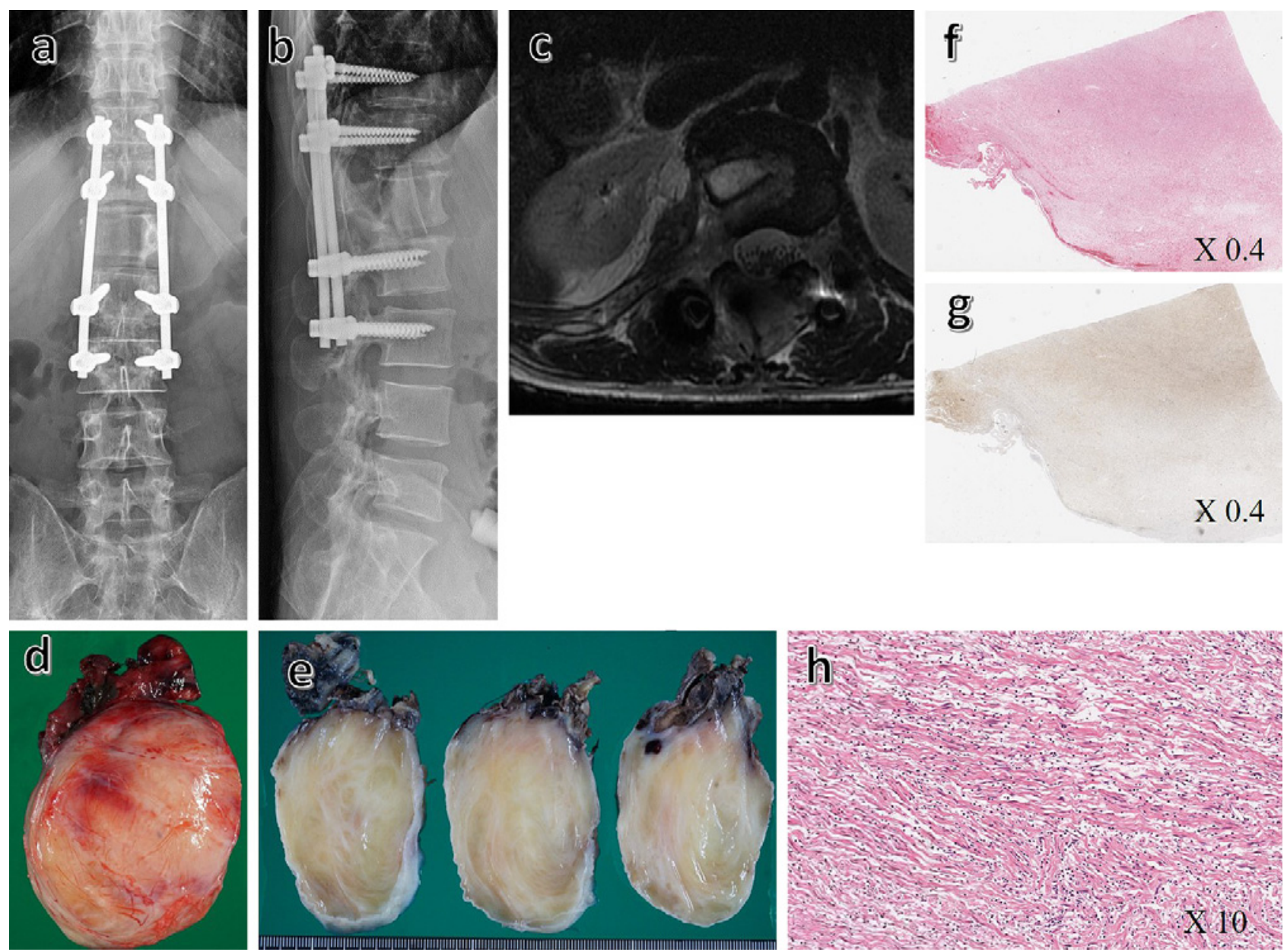

Figure 4. Tumor resection and T11-L3 posterior interbody fusion were performed for case 2. (a) Postoperative X-ray, coronal view. (b) Postoperative X-ray, lateral view. (c) Postoperative magnetic resonance imaging. T2-weighted axial image. No residual disease or local recurrence was found on MR images 4 months after the operation. (d) The resected tumor. The tumor was excised in one piece under the capsule. (e) On the cut section, the tumor presented as a uniform yellow-white solid mass without necrosis. (f) Hematoxylin and eosin staining, 0.4X magnification. (g) S-100 protein immunohistostaining, 0.4X magnification. (h) Hematoxylin and eosin staining, 10X magnification. The proliferation of wavy spindle cells with thickened collagen fiber bundles was observed; the tumor cells did not show any abnormal deformity or increased mitosis, and no tumor necrosis occurred. Immunohistochemical staining revealed that S-100 protein and SOX10 were partially positive and neurofilament was sporadic positive. Histopathological diagnosis was neurofibroma.

Spine Surgery and Related Research is an Open Access journal distributed under the Creative Commons Attribution-NonCommercial-NoDerivatives 4.0 International License. To view the details of this license, please visit (https://creativeco mmons.org/licenses/by-nc-nd/4.0/). 